\title{
EL PERSPECTIVISMO DE REINA DE AMÉRICA COMO CONOCIMIENTO SITUADO DE LA VIOLENCIA Y LA NECROPOLÍTICA EN EL PACÍFICO COLOMBIANO $^{1}$
}

\author{
POR \\ TANiA LiZARAZO \\ University of Maryland, Baltimore County
}

La literatura no puede salvarnos la vida. Con suerte, servirá para ayudarnos a resistir la derrota.

Nuria Amat, Reina de América

Escribo sobre la novela Reina de América en primera persona, en el exilio autoescogido que resulta ser la fórmula más cómoda para pensar Colombia. Y es que, ¿cómo pensar la violencia orgánica de esta nación en conflicto por más de cinco décadas si no es desde la paradoja de una primera persona que es en simultánea outsider? Mi interés no es hacer una crítica formal del uso de la primera persona en la prosa amatiana, ni tampoco un análisis exhaustivo de la representación del conflicto armado en la novela. Mi intención es establecer un espacio dialógico entre la construcción literaria amatiana de la violencia en el pacífico colombiano y las características sociales e históricas del conflicto en esta región: ¿Qué hace de Reina de América una narración efectiva y cómo las construcciones de género y raza dentro y fuera de la novela informan esta narración?

“HABLÉ DE TI ANTES DE QUE EXISTIERAS": CONOCIMIENTO SITUADO, FICCIÓN, METAFICCIÓN Y REALIDAD

En Reina de América, la narrativa sumerge a los protagonistas, Rat y Wilson, en una fantasía histórica que entreteje el erotismo, el hastío, la diversidad y la quietud del Chocó húmedo, una región olvidada por el estado colombiano, pero tan central para la ilegalidad de la coca, las masacres, las fumigaciones, la pobreza, y los desplazamientos. Todo incluido en la novela, de forma sutil, entre los silencios que marcan la interacción

\footnotetext{
1 Este artículo es un análisis derivado de una breve reseña publicada en la página web de Biblored, la red
} capital de bibliotecas públicas en Bogotá. 
entre Rat y Wilson; entre ellos y Aida: mujer, negra, bruja, víctima, victimaria; entre ellos y el contexto, la duda, la vida y la muerte. Para quien no ha ido a Chocó, las descripciones parecerán parte de un diario de viajes decimonónico: "la selva es un océano de espuma verde ennegrecida. Evito confundir el bosque con el cielo" (Amat 61). O al menos, una ficción poética.

Yo he estado en Chocó. Conozco la lluvia que no para: "imposible pensar en otra cosa que no sea el terco gotear del agua" (Amat 123), que adquiere un sentido más sagrado en una zona donde solo recientemente el agua empieza a lograr la connotación de servicio público. Las sensaciones de abandono, olvido y anacronía se confunden entre la angustia del querer vivir y la resignación ante la inmediatez de la guerra: "esto es la guerra de la guerra, dijo. Sólo hay un modo de comprender esta jodienda. No dijo cuál era porque habríamos tratado de considerar el método. Y si lo dijo, no recuerdo" (Amat 73). Entre la incertidumbre del abandono institucional y la cercanía de la guerra, la confusión y la vulnerabilidad reinan. Pero sobre todo, se impone una convicción fatalista e idealista: "tengo que hacer algo y no sé de qué se trata" (Amat 63).

La primera persona amatiana, así como los stream of consciousness de Rat pueden interpretarse como recursos feministas que privilegian la experiencia personal sobre la objetividad científica para dar cuenta del contexto del conflicto desde una perspectiva situada, directa y no mediada. Siguiendo a Haraway (1998), que propone los conocimientos situados como oposición a la autoridad (patriarcal) del conocimiento, considero que la narrativa situada de Amat logra un descentramiento de las estrategias de producción y recupera el plano experiencial en donde los conocimientos no solo se producen parcialmente sino además se viven parcialmente. Es así como la ficción amatiana puede leerse como un conocimiento del conflicto armado en el pacífico colombiano. El perspectivismo de Amat y, por tanto, de Rat, nos conduce al centro de producción de un conocimiento situado del conflicto armado colombiano, un conocimiento confiable en tanto parcial y ubicable. Igual que Bahía Negra es una ficción que se informa de la "realidad", Rat se informa de las experiencias personales de Nuria Amat. Así, la lectura nos sumerge en una historia personal, íntima, femenina y local, que deriva verdades sobre la experiencia nacional de la vida y la muerte en el pacífico colombiano, y por qué no, en Colombia, en general.

Leo a Amat y la leo en Rat: su relación con Óscar Collazos, su vida en Colombia. Me leo en Rat: mi condición de outsider en un área que conocí recientemente y mi privilegio racial, social y económico donde más del $90 \%$ de la población es negra y se encuentra la tasa de alfabetismo más baja del país. La figura de la catalana, la extranjera, es un símbolo intercambiable, un comodín de vulnerabilidad donde la propia identidad no es absoluta, en tanto que no es una narradora omnisciente ni omnipresente. Por el contrario, promueve un reconocimiento explícito del lugar de enunciación, la oportunidad de construcción de conocimientos situados (siguiendo a

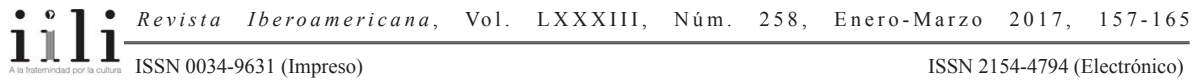


Haraway) y el perspectivismo de cada personaje: "tú, con tu narcisismo colombiano no puedes comprender la sensación de naufragio que me produce todo lo que pienso. Tu prepotencia española te hace olvidar lo que hicieron los españoles en América del Sur" (Amat 19). Este diálogo entre Wilson y Rat desestabiliza el lugar de enunciación y reconoce la importancia de la perspectiva en la interpretación del conflicto.

La historia se desarrolla en Bahía Negra, un lugar ficticio, caracterizado por la presencia permanente de una naturaleza indómita y atemorizante, un lugar rural y marginal a los productos materiales de las metanarrativas modernas de progreso y civilización. Bahía Negra nos recuerda a Macondo, un espacio abyecto desde la perspectiva de una nación centralista pero que resulta un lugar fundacional y representativo, un microcosmos de donde una realidad política y social más amplia puede derivarse. Y si en Macondo hay referencia a un hecho atroz como la masacre de las bananeras, no sorprende que en Bahía Negra también haya una masacre, que podría ser cualquiera de las dieciocho masacres que nombran los poemas de María Mercedes Carranza en El canto de las moscas, publicado en 1997. O cualquier otra de las 2.505 masacres que el Grupo de Memoria Histórica ${ }^{2}$ estableció como número aproximado para las matanzas masivas ocurridas entre 1982 y 2007 (11).

La falta de excepcionalidad de la violencia en Colombia puede ser una de las razones por las que los recursos literarios característicos de Nuria Amat, seguramente influenciados por el realismo mágico y el boom latinoamericano de los que ella ha aceptado recibir influencias, resultan efectivos en la creación de un mundo poético y verosímil para narrar lo innarrable: la violencia como vida cotidiana. Ésta no es una historia lineal con diálogos complejos entre los personajes. Es un flujo discontinuo, casi a manera de collage, donde se confunden la primera y la segunda persona constantemente. Es una novela vulnerable, de frases cortadas e indecisas que evocan la desesperanza del contexto de la guerra habitual en Colombia: "Nada importa. La noche es corta. Y ya estamos en otro día tan parecido al anterior como distinta es la noche de la espera. Se vive al momento. No se vive. La vida puede romperse en cualquier instante. Se respira con la intensidad propia de perdedores eternos. Ganar es morir, también, a veces" (Amat 59). La contradicción de la narrativa refleja los retos de la existencia en medio del conflicto, donde la supervivencia es ya un logro.

El objetivo de Nuria Amat no es crear una historia pulcra y pulida donde el lenguaje es invisible, sino precisamente cuestionar los alcances del lenguaje, la escritura, la

2 El grupo de Memoria Histórica fue creado como parte de la Comisión Nacional de Reparación y Reconciliación (CNRR) por la Ley 975 de 2005, durante el gobierno de Álvaro Uribe Vélez. Hacen parte de este grupo varios intelectuales famosos por sus investigaciones críticas sobre violencia, por las que fueron llamados "violentólogos". Ahora estos intelectuales de izquierda hacen parte de una iniciativa creada por un gobierno de derecha que nunca reconoció el conflicto y promovió leyes como la de "Justicia y Paz" que promueven reconciliación y reparación para una guerra que se cree acabada.

$111 \frac{\text { Revista Iberoamericana, Vol. LXXXIII, Núm. 258, Enero-Marzo 2017, }}{1157-165}$ 
literatura, para dar cuenta del horror. De ahí, tal vez, la inclusión de la escritura como actividad explícita en Rat y Wilson, que hablan sobre la dificultad y el peligro de escribir: "escribir es traicionar la lengua" (Amat 63). En algunas partes de la novela, estas reflexiones aparecen como elementos de metaficción. Como cuando se crea una relación urobórica entre la historia de amor que se narra y su existencia previa: "dijo Wilson que las historias de amor se escriben antes de ser vividas. Es decir, dijo, nuestro amor existió primero en forma de relato. Te inventé en mi cabeza. Hablé de ti antes de que existieras" (Amat 17). O cuando se hace referencia al papel escrito, a los textos, como envoltorios del horror: "el papel impreso valía bien poco si a la postre servía para envolver o limpiar las cosas más abyectas" (Amat 34). O a una novela dentro de la novela: "eres un escritor desesperado porque seguramente la novela que estás escribiendo es menos verdadera que tu vida" (Amat 129). O esta sentencia que puede aludir a la propia narradora: "Escribes como si fueses una muerta que recuerda" (Amat 158). El lenguaje es a la vez el medio y el obstáculo, la posibilidad de recordar y la imposibilidad de una memoria de los ausentes.

\section{RAZA Y GÉNERO EN El CONFLiCTO NECROPOlítico: “LA CATALANA DiCE QUE ESTAS CASAS LE RECUERDAN LOS BARRACONES NAZIS"}

El cuestionamiento interno de la novela y su narradora como unidades imperturbables permite establecer una conexión más dinámica con la autora y su obra. Al mismo tiempo, la caracterización de la propia narradora y su experiencia en términos de "otredad" por la no pertenencia también da lugar a comparaciones históricas: "la catalana dice que estas casas le recuerdan los barracones nazis" (Amat 33). Además del carácter cómico de esta frase dentro del contexto específico en que se enuncia, es también conveniente recordar que el Holocausto, en estudios de la memoria, ha sido un tropo que permite darle valor histórico a hechos violentos que sin tal comparación carecerían de resonancia internacional. Esto es útil para establecer la equivalencia entre hechos violentos, aunque también establece una jerarquía occidentalizada donde los hechos particulares son carentes de valor en sí mismos. El horror requiere verosimilitud, pero en medio del horror la paranoia y la duda reinan: "Me resulta imposible creer en lo que dices del mismo modo que me resulta imposible no dejar de pensar en lo que dices" (Amat 115). Que la narradora sea la "otra" crea una duda implícita que la primera persona atenúa al crear una conexión afectiva con la narradora y sus experiencias.

Rat es extranjera, es blanca, y escoge sumergirse en la violencia por su cuenta. Así se establece como la antítesis de Aida, una mujer que nace en medio del conflicto y está marcada por lo peor de él: el incesto, el duelo, la venganza. Aida encarna las observaciones de Beatriz Preciado sobre la construcción colonial de los cuerpos femeninos negros como cuerpos monstruosos, incontrolables, salvajes, que son marcados como anormales y expuestos como ejemplarizantes de una cultura o la falta de ésta:

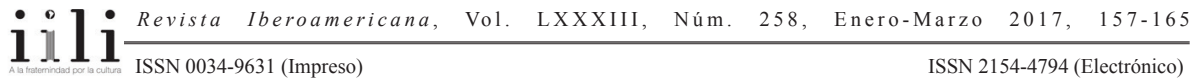


el cuerpo físico de las mujeres de color, auténtica metáfora orgánica en el intercambio discursivo y material de la globalización, se ve al mismo tiempo invisibilizado y sobre-expuesto a los dispositivos visuales de control y mediatización, hecho público a través de técnicas de representación que impiden, más que garantizan, el acceso al espacio público y que exigen la creación de espacios públicos subalternos de resistencia. (Preciado 14-5)

Pero aunque Aida pudiera pensarse como la ejemplificación ideal de la abyección kristeviana, la abyección como parte necesaria de la construcción de identidad en oposición a un "otro" rechazado, implica que no existe nada ontológicamente abyecto, sino que algo es desterrado a los límites de lo considerado "humanamente" inteligible como reafirmación de una identidad (nacional, de clase, etc.) ontológicamente excluyente que lo marca como "abyecto". Lo "abyecto" no es entonces más que lo excluido por el poder hegemónico, una muerte ontológica del cuerpo negro en relación con la sociedad civil, según el afropesimismo, al considerar que la posición de los negros frente al resto de la humanidad es un antagonismo estructural y no simplemente un conflicto. De esta corriente filosófica se deriva la propuesta del filósofo camerunés Achille Mbembe en su famoso artículo "Necropolitics", donde redefine la noción de soberanía para ligarla no solo al ejercicio del poder sobre la vida como en Foucault, sino al de la muerte.

Según mi lectura de Mbembe, en estados de excepción, donde se suspenden temporalmente los derechos de los ciudadanos, la raza funciona como reguladora de la distribución de la muerte. Este ejercicio del poder sobre la muerte, dentro de un marco de jerarquía racial, es tomado por los ciudadanos por su propia mano ante la ausencia de un orden institucional controlado por el estado. Es así como podría entenderse que el sistema en funcionamiento en Colombia no es un gobierno democrático, sino una colonia tardía donde la guerra es la única ley, según la definición de Mbembe:

in modern philosophical thought and European political practice and imaginary, the colony represents the site where sovereignty consists fundamentally in the exercise of a power outside the law (ab legibus solutus) and where "peace" is more likely to take on the face of a "war without end". (13)

Esta guerra sin fin que caracteriza a la colonia, nos lleva de vuelta a Aida, que no puede escapar al ciclo de la violencia que la transforma de víctima a victimaria. Esto puede interpretarse como una forma de resistencia causada por la opresión asociada a su cuerpo negro, como en la cita de Preciado. Una forma de trasgredir un espacio culturalmente dominado por hombres y que, por su militarización, promueve la masculinización de la vida. Amat nota la trasgresión del género como la mejor posibilidad de existencia: "Descubrí que en este lugar era más fácil ser filósofa o diablesa que una mujer cualquiera" (94). En el mismo sentido, la india Lucila como

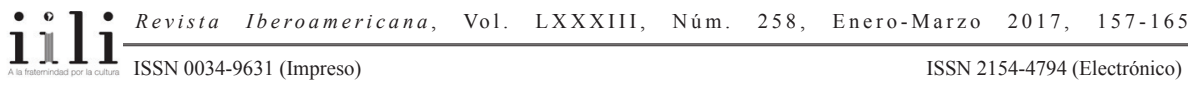


mujer subversiva encarna también la peligrosidad de la resistencia, que es también la única forma de agencia ante la exclusión: "acá la lengua no puede volar sola y a su aire sin que provoque temor y presentimientos aciagos. $\mathrm{Y}$ el miedo enciende el cerebro de lo prohibido. No hable tanto. Hágame el favor" (112). Y ya sabemos que la imposibilidad de dejar de hablar, implica que alguien ejercerá por su propia mano el derecho de ejecutar a la que no puede callarse, con más justificación de la jerarquía necropolítica si es mujer y no es blanca.

\section{“SENTÍ QUE ESTA MUERTE NO ME PARECÍA TAN ESPANTOSA": LA VIOLENCIA COMO COTIDIANIDAD}

Las ejecuciones ilegales características del régimen necropolítico aparecen permanentemente en Reina de América sin ninguna justificación más allá que la decisión de un grupo, un combatiente o una exvíctima solo para confirmar la propia existencia: "en el fondo nadie sabe. Ni se sabrá nunca. Se mata por puras ganas de vivir. Para no ser el primero en ser el muerto" (Amat 15). Se mata sin razón. Se mata porque los demás se están matando y porque es la única forma de alejar temporalmente el miedo, la muerte. Según Mbembe, matar está estrechamente ligado a la supervivencia del horror: "the logic of survival one's horror at the sight of death turns into satisfaction that it is someone else who is dead. It is the death of the other, his or her physical presence as a corpse, that makes the survivor feel unique. And each enemy killed makes the survivor feel more secure" (36). La supervivencia no puede desligarse de la muerte del "otro", de la eliminación de lo que amenaza la propia existencia.

Pero la lógica de matar por supervivencia es inentendible para quien no se encuentra absorto por la paranoia de la violencia: "es de locos, pensé. Ni los asesinos saben por qué matan. El monte olía a muerte a nuestro alrededor" (Amat 81). El carácter sinestésico con el que Rat describe la muerte evidencia la imposibilidad de separación consciente de la guerra. La violencia implica respuestas emocionales que interrumpen la percepción de los espacios y la vida misma: "entonces toda la tristeza del mundo me caía encima como presagio de que algo horrible iba a sucedernos. El miedo no se deja ver. Se siente pero no se escucha" (Amat 30). Pero a pesar del trauma y el horror, la vida continúa. Y es tal vez esto lo más difícil para Rat, para Nuria, para mí, y tal vez para cualquiera que piense críticamente la violencia: "Ya no soy capaz de ver en el horizonte de la vida la marca del horror que sigue sucediendo. Esto es lo más difícil de sobrellevar. La ceguera" (Amat 125). La ceguera es la normalización de la violencia cuando la muerte es inseparable de la vida cotidiana, como en el pacífico colombiano.

Sobrevivir la guerra, seguir viviendo en medio de su influjo, genera desensibilización por el contacto extendido con el efecto anestesiante de la violencia: "sentí que esta muerte no me parecía tan espantosa. Wilson estaba muerto. Cornelia muerta. También habían matado a la maestra, al cartero, al alcalde y a decenas de campesinos inocentes.

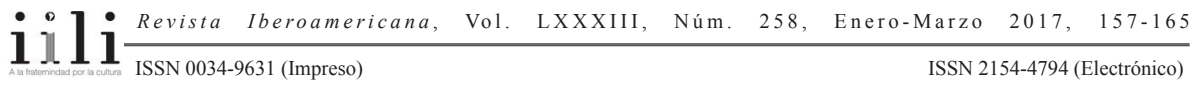


Lo más horrible era pensar que Aida y yo seguíamos vivas" (Amat 220). La propia existencia se subestima ante la inminencia de "otros" inocentes asesinados, lo que marca el conflicto doblemente por su horror: el horror de la muerte se suma al horror de vivir para contarla. Este ciclo imparable de muerte y duelo no es exclusivo de la historia que narra Nuria, sino la experiencia de más de 5 millones de personas desplazadas internamente en Colombia ${ }^{3}$ y las incontables víctimas invisibilizadas por la hipótesis ampliamente difundida dentro y fuera del país de que el conflicto se ha terminado.

La idea de que Colombia es una nación en posconflicto fue promovida por el gobierno de Álvaro Uribe Vélez (2002-2010) y su sucesor Juan Manuel Santos, y ha sido fortalecida por los recientes asesinatos de Carlos Castaño, líder paramilitar de las AUC (Autodefensas Unidas de Colombia), en 2004 y los líderes de las FARC (Fuerzas Armadas Revolucionarias de Colombia) Manuel Marulanda en 2008 y Alfonso Cano en 2011. Sin embargo, la penetración del conflicto en la cotidianidad ha permitido su renovación constante, lo que hace que la novela de Amat refleje el estado actual del conflicto armado en Colombia. Es bastante ilustradora su visión simplista del conflicto como una guerra "por nada" donde los perjudicados son siempre los campesinos: "cuando la gente se pelea por nada, dice, ninguno lleva la razón. Si de veras se desea la paz, resulta difícil tomar partido por uno u otro bando. Como persona, como hombre, como colombiano siempre estaré de parte del pueblo, del lado del campesino, que es al que terminan matando" (Amat 13).

No creo que mucho haya cambiado desde la publicación de Reina de América. Colombia sigue siendo el país más peligroso para ser sindicalista, periodista y, en general, parte de una minoría: mujer, indígena, negra o campesina. Es el país del hemisferio occidental que más recibe ayuda militar de Estados Unidos y, tal vez por eso, también el país con mayor número de violaciones de derechos humanos. La violencia no se ejerce solo ilegalmente por guerrillas y grupos paramilitares, sino que se desenvuelve como un continuum al que se suma el ejército y la policía no como defensores de los derechos civiles, sino también como perpetadores, con sus propios intereses políticos y económicos, que muchas veces son compatibles con los de los grupos ilegales. Amat lo describe perfectamente desde la perspectiva de los campesinos:

Los campesinos, siguió diciendo, estamos a merced de los saboteadores de tierras. De una parte, somos esclavos de los narcotraficantes y de la guerrilla, dos enemigos en dos frentes opuestos. Y cuando al Ejército le da la ventolera de enviar la policía de Quibdó para quemar y fumigar nuestras plantaciones somos también nosotros los que perdemos. (133)

3 Dato tomado de la última actualización en septiembre del año 2015 del "Internal Displacement Monitoring Centre", lo que ubica a Colombia como el primer país con más desplazados en el mundo.

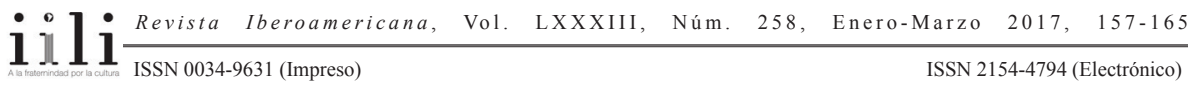


La continuidad de la guerra contra los campesinos colombianos, contra los que pelean sin distinción los ejércitos legales e ilegales impulsados por la plata del narcotráfico es descrita también por Michael Taussig, en su libro My Cocaine Museum: "Behind the guerrilla come the paramilitaries. And they, too, depend on drugs shipped statewise. The guerrilla can be tough on civilians, the paras even more so, spectacular massacres of defenseless peasants being their stock in trade" (146). Por eso, el final trágico de Wilson, su tío y su prima, y todos los demás que asistían al "baile de la coca" no sorprende. Ni siquiera a Rat. Y ni aún a un lector desprevenido. Como no sorprenderán tampoco los titulares de nuevas masacres en pueblos remotos de Colombia donde nunca han ido ni irán los colombianos "de bien" que marchan en contra de los secuestrados de las FARC, pero siguen apoyando la hipótesis del gobierno de turno de que el conflicto ya no existe y las FARC son un grupo anacrónico que debe desaparecer para lograr la tan anhelada paz.

\section{CONCLUSIÓn: "Un SILENCIO. No RESPIRO"}

El tema de la violencia ha creado una nueva demanda y, por tanto, un nuevo mercado para la industria editorial, que incluye desde memorias de exsecuestrados, guerrilleros o paramilitares hasta crónicas periodísticas o novelas de ficción sobre la imparable guerra en Colombia. Con esta sobreproducción de los últimos años parecería que una novela escrita por una catalana no tendría nada que aportar. Sin embargo, la lectura personal y distante que hace Nuria Amat permite leer el conflicto desde la intensidad de sus silencios y el pastiche de sus descripciones y diálogos. Una vez más, la potencia de Reina de América reside en la presentación de las verdades más terribles como la presencia innegable de la muerte que no es nada si se compara con el dolor de poder vivir tras la muerte del amante:

más que un hombro quiero una voz a mi lado. Un nido de palabras. Un silencio. No respiro. Moriré de amor. Moriré de inanición. Si no respiro, moriré. Si soy picada por cierto mosquito, moriré también. Si el hongo herbicida es el llamado fusarium oxysporum moriré también. Si muere Wilson, moriré. (64)

No morir se presenta como el peor castigo porque implica el destierro del desplazamiento. Rat se vuelve una desplazada más, o una migrante, si queremos usar el eufemismo políticamente correcto con el que se ha nombrado la tragedia del desarraigo, el duelo de la supervivencia. Nuria no dice nada nuevo, nada que no hayan dicho las investigaciones o los medios, como Rat no vive nada que no haya vivido una campesina chocoana, pero su escritura desde la distancia, nos envuelve en una pausa, nos devuelve al "tengo que hacer algo y no sé de qué se trata" porque así como

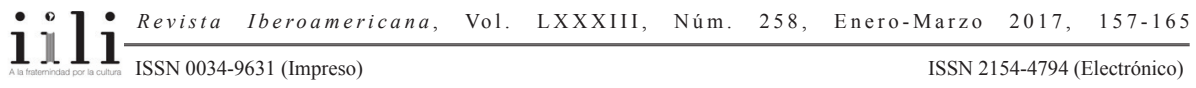


al final Rat "no tenía nada en las manos más que un nombre. Wilson Cervantes. Y un silencio" (Amat 237), ¿qué se puede ofrecer que sea más contundente que un silencio?

\section{OBRAS CITADAS}

Amat, Nuria. Reina de América. Barcelona: Seix Barral, 2002.

Carranza, María Mercedes. "El canto de las moscas. Versión de los acontecimientos". Golpe de dados. Revista de poesía 35/50 (nov.-dic. 1997).

Grupo de Memoria Histórica. Trujillo, una tragedia que no cesa: Primer informe de Memoria Histórica de la Comisión Nacional de Reparación y Reconciliación. Bogotá: Planeta, 2008.

Haraway, Donna. "Situated Knowledges: The Science Question in Feminism and the Privilege of Partial Perspective." Feminist Studies 143 (Fall, 1988): 575-99.

Mbembe, Achille. "Necropolitics.” Public Culture 15/1 (2003): 11-40.

Preciado, Beatriz. "Gigantas / casas / ciudades. Apuntes para una topografía política del género y la raza". Artecontexto. Arte, cultura y nuevos medios 8(Otoño 2005): 8-21. Taussig, Michael. My Cocaine Museum. Chicago: U of Chicago P, 2004. 
